\title{
Projet d'étude des stratégies de reproduction des populations sahéliennes à partir de l'enquête « Dynamique migratoire, insertion urbaine et environnement au Burkina Faso » A RESEARCH PROJECT ON THE REPRODUCTIVE STRATEGIES OF POPULATIONS IN THE SAHEL BASED ON THE SURVEY "MIGRATION DYNAMICS, URBAN INTEGRATION AND ENVIRONMENT IN BURKINA FASO”
}

\author{
Jean Poirier, Victor Piché, Gaël Le Jeune, Bonayi Dabiré et Hamdou Rabby \\ Wane
}

Volume 30, numéro 2, automne 2001

Transition démographique et urbanisation au Québec à la fin du XIX siècle et au début du $\mathrm{XX}^{\mathrm{e}}$ siècle

URI : https://id.erudit.org/iderudit/010312ar

DOI : https://doi.org/10.7202/010312ar

Aller au sommaire du numéro

Éditeur(s)

Association des démographes du Québec

ISSN

0380-1721 (imprimé)

1705-1495 (numérique)

Découvrir la revue

Citer cet article

Poirier, J., Piché, V., Le Jeune, G., Dabiré, B. \& Wane, H. R. (2001). Projet d'étude des stratégies de reproduction des populations sahéliennes à partir de l'enquête « Dynamique migratoire, insertion urbaine et environnement au Burkina Faso ». Cahiers québécois de démographie, 30(2), 289-309.

https://doi.org/10.7202/010312ar
Résumé de l'article

Cette note présente l'enquête nationale « Dynamique migratoire, insertion urbaine et environnement au Burkina Faso ", réalisée conjointement en 2000-2001 par le Département de démographie de l'Université de Montréal, le CERPOD et l'UERD dans le cadre du Programme population et développement au Sahel. Cette enquête vise une meilleure compréhension des stratégies de reproduction que mettent en oeuvre les populations du Burkina Faso. Une première partie situe l'enquête sur le plan théorique en illustrant les différentes facettes du concept de stratégie de reproduction à l'aide d'exemples tirés de travaux portant sur le Burkina Faso. La seconde partie présente les objectifs et le plan de sondage de l'enquête et décrit l'information collectée à chacun des niveaux d'analyse : individu, ménage, communauté, région et pays Pour chaque niveau, nous insistons sur les défis de la collecte, les innovations de notre enquête et son potentiel en matière d'analyse, en soulignant certaines hypothèses importantes que nous comptons tester. 
Cahiers québécois de démographie

Vol. 30, no 2, automne 2001, p. 289-309.

\title{
Projet d'étude des stratégies de reproduction des populations sahéliennes à partir de l'enquête " Dynamique migratoire, insertion urbaine et environnement au Burkina Faso "
}

\author{
Jean POIRIER *, Victor PICHÉ *, Gaël LE JEUNE *, \\ Bonayi DABIRE † et Hamdou Rabby WANE :
}

Cette note présente l'enquête nationale "Dynamique migratoire, insertion urbaine et environnement au Burkina Faso ", réalisée conjointement en 2000-2001 par le Département de démographie de l'Université de Montréal, le CERPOD et l'UERD dans le cadre du Programme population et développement au Sahel. Cette enquête vise une meilleure compréhension des stratégies de reproduction que mettent en ceuvre les populations du Burkina Faso. Une première partie situe l'enquête sur le plan théorique en illustrant les différentes facettes du concept de stratégie de reproduction à l'aide d'exemples tirés de travaux portant sur le Burkina Faso. La seconde partie présente les objectifs et le plan de sondage de l'enquête et décrit l'information collectée à chacun des niveaux d'analyse : individu, ménage, communauté, région et pays. Pour chaque niveau, nous insistons sur les défis de la collecte, les innovations de notre enquête et son potentiel en matière d'analyse, en soulignant certaines hypothèses importantes que nous comptons tester. English abstract, p. 309.

T es avancées récentes dans les méthodes d'analyse multiLniveaux des transitions ouvrent de nouvelles perspectives stimulantes dans de nombreux domaines en sciences sociales, dont celui de l'étude des stratégies de reproduction des populations. En effet, en permettant d'enrichir la description et la comprêhension de processus sociaux complexes, ces développements méthodologiques ne manqueront pas d'alimenter la réflexion théorique. Cependant, pour que ces promesses soient

* Département de démographie, Université de Montréal. † Unitẻ d'enseignement et de recherche en démographie, Université de Ouagadougou, Burkina Faso. ₹ Programme majeur population et développement (CERPOD) de 1'nstitut du Sahel, Mali. 
tenues, les nombreux défis que pose la collecte de données adéquates pour l'utilisation de ces méthodes devront être relevés.

L'objectif de cette note est d'illustrer le potentiel de ces méthodes et les défis qu'elles soulèvent à partir de l'expérience de l'enquête "Dynamique migratoire, insertion urbaine et environnement au Burkina Faso ". Il s'agit d'une vaste enquête nationale réalisée conjointement par de Département de démographie de l'Université de Montréal, le CERPOD et l'UERD dans le cadre du Programme population et developpement au Sahel. L'enquête vise une meilleure compréhension des stratégies de reproduction que mettent en œuvre les populations du Burkina Faso, en vue d'éclairer la prise de décisions.

Cette note comporte deux grandes parties. La première présente le concept de stratégie de reproduction, dont l'étude constitue l'objectif général de notre recherche. Nous situons ce concept sur le plan théorique en illustrant ses différentes facettes à l'aide d'exemples tirés de travaux portant sur le Burkina Faso. Cela nous permet du même coup de présenter des caractéristiques importantes du pays que nous étudions. En conclusion de cette première partie, nous esquissons les grandes lignes de l'approche qui sous-tend notre choix d'utiliser les méthodes d'analyse multi-niveaux des transitions et qui a guidé nos décisions en matière de collecte de données.

Dans la seconde partie de cette note, après avoir présenté les objectifs et le plan de sondage de l'enquête, nous décrivons l'information collectée à chacun des niveaux d'analyse : indivi$\mathrm{du}$, ménage, communauté, région et pays. Pour chaque niveau, nous insistons sur les défis de la collecte, les innovations de notre enquête et son potentiel en matière d'analyse en soulignant certaines hypothèses importantes que nous comptons tester.

\section{LES STRATÉGIES DE REPRODUCTION À LA LUMIËRE DES DÉBATS THÉORIQUES ET DE LA RÉALITÉ BURKINABÉ}

Au cours des vingt-cinq dernières années, le concept de stratégies de reproduction s'est imposé graduellement en démographie comme dans les autres branches des sciences sociales. Ce concept vise à rendre compte de l'enchainement de comportements, démographiques mais également économiques, sociaux et autres, qu'adoptent les individus et les ménages pour attein- 
dre certains objectifs qui vont du maintien de leurs moyens d'existence, de leur niveau de vie et de leur statut social à l'accumulation et à la mobilité ascendante.

Initialement développé au début des années soixante-dix pour mieux comprendre les comportements des populations du Tiers Monde (Bourdieu, 1994), le concept de stratégies de reproduction s'est graduellement imposé en sciences sociales avec le développement des approches biographiques (" lifecourse approaches ") (Moen et Wethington, 1992; Upchurch, Lillard et Panis, 1995). Il constitue aujourd'hui un outil indispensable pour l'analyse des réponses des populations du Tiers Monde aux situations de crise, tant en milieu rural qu'en milieu urbain.

Cette popularité s'explique selon nous par quatre raisons principales.

Premièrement, ce concept permet d'étudier les processus sociaux, les comportements collectifs, non pas de façon isolée mais en interrelation les uns avec les autres. Cet avantage est particulièrement appréciable en démographie, science qui avait évacué le problème de l'interaction "en traitant chaque phénomène séparément, se contentant d'éliminer l'effet des autres phénomènes considérés comme perturbateurs " (Courgeau et Lelièvre, 1993: 23). Il ouvre le champ à l'étude de ce que Blossfeld (1996) qualifie de processus sociaux parallèles et permet ainsi d'affiner l'analyse causale.

Pour le Burkina Faso, plusieurs travaux qui s'inscrivent dans des champs disciplinaires différents (anthropologie, démographie, économie rurale, géographie) soutiennent cette hypothèse d'imbrication et d'interaction des processus sociaux. Certains d'entre eux sont centrés sur les liens qu'entretient la migration, historiquement fort importante au Burkina, avec la nuptialité d'une part et l'activité d'autre part. Par exemple, dans le contexte du contrôle exercé par les ainés sur la terre et les épouses, qui caractérise l'essentiel du milieu rural au Burkina, la migration des jeunes hommes célibataires (les " cadets ") en Côte-d'Tvoire serait une étape presque obligée, au moins dans certaines régions, dans leur accès à l'indépendance économique (Skinner, 1965; Deniel, 1968; Boutillier, 1975).

D'autres travaux ont souligné que les mariages s'inscrivent encore souvent en milieu rural dans une perspective d'échange et d'établissement d'alliances, ces dernières constituant notamment des filets de sécurité sur lesquels s'appuyer en cas de besoin. Pour accroître l'efficacité de tels mécanismes, les aînés 
chercheraient alors à marier certaines de "leurs " filles dans un village qui n'appartient pas à la même zone agro-écologique et qui serait alors moins vulnérable en cas d'accident climatique. La constitution de telles alliances entrainerait ainsi la mise en place de réseaux migratoires autour de la circulation des femmes, des enfants... (Reardon, Matlon et Delgado, 1988).

Pour plusieurs chercheurs, la migration est partie prenante des efforts de diversification des activités économiques des membres des ménages ruraux face aux risques élevés que présente le seul recours à l'agriculture. La migration rurale-rurale s'expliquerait alors par la pratique d'activités d'élevage, de petit commerce, de culture de saison sèche, d'orpaillage... La migration internationale accompagnerait ainsi la recherche de travail sur les plantations de café et de cacao en Côte-d'Tvoire ou la pratique d'activités d'élevage dans les pays côtiers limitrophes du sud, Bénin, Togo, Ghana. La migration rurale-urbaine s'inscrirait quant à elle dans la recherche d'emplois rémunérés en milieu urbain, essentiellement dans le secteur informel.

Les liens entre nuptialité, fécondité et production agricole ont également été abordés dans différents travaux. Pour certains chercheurs, la polygynie jouerait un rôle crucial dans la capacité de mise en culture des terres (Singh, 1988). Elle permettrait d'une part d'accéder à une force de travail indispensable d'usage immédiat, mais également, par les enfants auxquels les femmes donnent naissance, d'accumuler une force de travail d'usage différé (Izard, 1985). En effet, les enfants de moins de 15 ans ont des journées plus chargées que les personnes âgées (Ancey, 1983). Le travail des enfants, qui est en outre essentiel à l'élevage, est une source de revenu indispensable pour faire face à l'insécurité alimentaire (Sawadogo et Larivière, 1993; Singh, 1988).

Deuxièmement, le concept de stratégie de reproduction permet de prendre en considération le rôle important des facteurs de niveau macro dans l'explication des comportements individuels. La reconnaissance de ce rôle est au centre d'une des traditions théoriques influentes en sciences sociales (Piché et Poirier, 1990 et 1995). En limitant le répertoire des options disponibles à chaque moment aux individus, les structures sociales non seulement façonnent leurs stratégies mais également déterminent qui sera en mesure de tirer le plus grand profit de telle ou telle stratégie.

Se pose alors le problème des structures les plus influentes et de leur articulation. Selon l'approche théorique privilégiée, 
l'accent est mis sur le rôle déterminant de tel ou tel ensemble de structures macro-sociales.

Les structures "agro-écologiques " sont au centre des approches en termes d'éco-types ou d'éco-systèmes développées par les anthropologues scandinaves (Mitterauer, 1992). Au Burkina Faso, plusieurs travaux ont attiré l'attention sur le poids de l'environnement sur les comportements individuels. Deux points ressortent clairement de ces travaux. Premièrement, la diversité de l'environnement au Burkina Faso. Selon les critères retenus, on distingue de trois à cinq zones "agroécologiques" en milieu rural qui regroupent 85 pour cent de la population. Ces zones présentent de grands écarts en termes de densité et de croissance démographique. Quant à la zone urbaine, elle se structure d'une part autour de deux villes principales, Ouagadougou, la capitale, et Bobo Dioulasso; et d'autre part autour d'une série de petits centres dont certains connaissent une croissance rapide, comme Banfora. Le second point concerne l'ampleur des risques que font peser sur les populations ces différents types d'environnement. En milieu rural burkinabè, les populations tirent l'essentiel de leur subsistance de l'agriculture pluviale et de l'élevage. Les conditions pédologiques défavorables qui caractérisent la plus grande partie du pays et la grande variabilité interannuelle et spatiale de la pluviométrie imposent de lourdes contraintes aux populations (Poirier et Guiella, 2000).

C'est dans ce contexte que Hampshire et Randall (1999) ont mis en évidence le rôle de la migration saisonnière pour les populations du Sahel burkinabè. Elles constatent que les couches les plus défavorisées de ces populations ne participent pas aux migrations saisonnières, contrairement aux couches les plus riches, qui en tirent le plus grand bénéfice. De ce fait, la migration saisonnière tendrait à accroître les inégalités au sein de ces populations.

Les approches d'économie politique (ou matérialistes ou historico-structurelles) insistent sur le rôle déterminant des structures de classes découlant du processus de développement du capitalisme pour expliquer les comportements démographiques. Au Burkina, plusieurs travaux ont documenté l'impact des politiques coloniales et néo-coloniales sur l'évolution de la population en général (Gervais, 1990), plus particulièrement sur la mise en place d'un système de migration circulaire (Cordell, Gregory et Piché, 1996). 
Les structures culturelles ou normatives liées aux modes d'organisation sociale sont au centre de certaines approches de la modernisation. Pool et Coulibaly (1970) ont ainsi attribué la stabilité des forts niveaux de fécondité des populations rurales du Burkina à l'influence des structures socioculturelles. Cette influence devait se diluer avec le processus d'urbanisation rapide que connaissait alors le pays. Des travaux plus récents (Kevane et Wydick, 2001) illustrent l'effet différentiel de ces normes sur l'allocation du temps de travail des femmes dans différents groupes ethniques du Burkina.

Les structures patriarcales découlant des rapports de genre sont au cœur des approches féministes. Au Burkina Faso, plusieurs travaux ont mis en évidence l'influence de ces structures sur la situation des femmes (Kevane et Gray, 1999). Guiella et Poirier (2000) soulignent ainsi qu'en pays Mossi les femmes font face à de très fortes contraintes économiques. Leur statut foncier est précaire. Elles n'ont pas de contrôle sur la distribution des terres et l'attribution d'un champ personnel est le fait du mari après quelques années de mariage.

À ces contraintes auxquelles doivent faire face toutes les femmes s'ajoute pour les jeunes mariées une situation sociale très précaire que Gruenais $(1985: 238)$ résume ainsi : "Instabilité, faible intégration dans la famille du mari, soumission totale aux "mères" du mari, dépossession de leurs enfants".

Plus récemment, des chercheurs ont également pris en compte des structures qui opèrent à un niveau intermédiaire, entre le global et l'individuel, entre le macro et le micro. Ces approches " institutionnelles " centrent l'attention sur l'influence potentielles des structures foncières, des infrastructures communautaires, des églises, de l'environnement domestique etc. sur les comportements socio-démographiques.

Ainsi, l'étude du peuplement des zones libérées de l'onchocercose au Burkina Faso a bien mis en évidence le rôle déterminant des axes de communication dans la réussite ou l'échec de l'implantation des migrants dans de nouvelles régions du pays (McMillan et al., 1998). De même, Laurent (1996) démontre l'impact important de la pénétration des Églises protestantes sur la transformation de l'institution matrimoniale dans certaines zones du Burkina. Enfin, pour Saint-Pierre, Gregory et Simmons (1986), la structure des ménages doit être prise en compte dans l'explication des comportements migratoires des Burkinabè. 
En troisième lieu, le concept de stratégie de reproduction permet de reconnaitre clairement le choix rationnel des acteurs dans les limites qu'imposent les structures sociales. Dans cette perspective influente en sciences sociales, les acteurs sociaux agissent intentionnellement et les comportements sont orientés en fonction d'objectifs poursuivis. La chaine causale doit donc prendre en compte le "libre arbitre" des individus. Pour des chercheurs (Blossfeld et al., $1999: 230$ ) :

It seems agreed that demographic phenomena are always directly or indirectly based on actions of individuals. Demography does not deal with associations among variables per se but with variabies that are associated via acting people.

Ces actions constituent non seulement des réponses aux structures en place mais elles contribuent également à les modifier. Elles peuvent être de nature préventive, visant à minimiser les risques et à éviter une crise, ou de nature adaptative, c'est-à-dire répondre à une situation de crise pour en atténuer les effets ou en limiter la durée.

Ainsi, face aux épisodes récurrents de sécheresse liées à la grande variabilité interannuelle de la pluviométrie, les populations rurales du Burkina Faso adoptent plusieurs mesures de minimisation des risques de famine et d'insécurité alimentaire : diversification régionale des activités agricoles, diversification des activités économiques hors de l'agriculture, migration vers les villes ou en Côte-d'Ivoire... (Reardon, Matlon et Delgado, 1988).

En milieu urbain, Sauerborn et al. (1996) ont montré qu'à Ouagadougou, la capitale du Burkina, tout un ensemble de comportements de différents types permettait de réagir à la crise provoquée par un événement comme la maladie ou la mort d'un membre du ménage : réduction de la consommation alimentaire, retrait des enfants de l'école, pratique d'une activité secondaire, vente d'une partie du patrimoine, etc.

La quatrième caractéristique théorique intéressante du concept de stratégie de reproduction est son dynamisme, c'est-àdire qu'il intègre forcément la dimension temporelle. Les pratiques sur lesquelles s'appuient les stratégies s'enchaînent en effet de façon chronologique.

Au Burkina Faso, cette perspective temporelle apparait clairement pour la migration, le système de migration circulaire étant forcément dynamique (Cordell, Gregory et Piché, 1996). Il en va de même pour la nuptialité. Laurent (1996) souligne ainsi que dans l'organisation sociale Mossi, l'échange des femmes 
repose avant tout sur des relations entre lignages qui s'instaurent sur une longue période (au moins trois générations). Enfin, l'alternance d'une courte saison des pluies et d'une longue saison sèche, caractéristique du climat tropical au Burkina, exige forcément l'enchaînement chronologique des activités de production en milieu rural.

On le devine aisément, l'opérationnalisation du concept de stratégies de reproduction dans les quatre dimensions que nous venons de présenter et d'illustrer à partir d'exemples burkinabè représente un défi considérable. Des avancées méthodologiques récentes sur le plan du traitement, de l'analyse et de la collecte des données nous permettent de nous y attaquer.

En effet, à partir de la fin des années cinquante, la généralisation du recours à l'ordinateur pour le traitement de larges bases de données accélère la reconnaissance du potentiel des histoires de vie comme source privilégièe d'information en sciences sociales. Les biographies perdent rapidement leur étiquette de "soft data " pour faire leur entrée dans le domaine de la démographie et de la sociologie quantitative. Mais pendant longtemps, les grandes opérations de collecte ne portent que sur un seul domaine de la biographie des individus. Pour les pays en développement, il s'agit bien sûr le plus souvent de l'histoire génésique, compte tenu du contexte idéologique en vigueur au cours des années soixante et soixante-dix (Piché et Poirier, 1990). Si les grands programmes des Enquêtes mondiales sur la fécondité ${ }^{1}$ et des Enquêtes démographiques et de santé $^{2}$ vont permettre des avancées importantes sur le plan de la description du phénomène, leur bilan sur le plan explicatif demeure fort mitigé. Dès 1969, des chercheurs attiraient d'ailleurs l'attention sur les limites inhérentes aux données ne portant que sur une seule trajectoire des individus (Balan et al., 1969: 106). En ce qui concerne l'explication des comportements démographiques, Poirier, Piché et Neill (1989), pour la fécondité, et Lelièvre (1999), pour la migration, ont fait écho à ces critiques.

Les progrès dans le domaine de la modélisation vont permettre, dans un premier temps, de dépasser ces limites et d'entreprendre l'étude de l'articulation dynamique des trajec-

Les Enquêtes mondiales sur la fécondité ont été réalisées entre 1974 et 1983 dans divers pays en développement.

2 Les Enquêtes démographiques et de santé ont commencé en 1985 au Salvador et se poursuivent jusqu'à présent (on prévoit une enquête en 2002 à Madagascar; pour ce pays, il s'agit d'un troisième passage). 
toires dans la vie des individus (Lelièvre, 1999; Upchurch, Lillard et Panis, 1995).

Ils vont ensuite permettre de prendre en compte, dans l'explication des comportements individuels, des caracteristiques mesurées à différents niveaux d'agrégation (Courgeau et Baccaïni, 1997). Mais, comme le soulignent Courgeau et Baccaïni (1997: 860), l'utilisation de tels modèles biographiques multi-niveaux se heurte trop souvent à des problèmes d'observation et de données disponibles.

L'étude empirique des stratégies de reproduction exige en effet des données qui doivent permettre de :

1. Reconstituer les trajectoires des individus "dans des domaines variés qui interagissent "(Lelièvre, $1997: 197)$ de façon à pouvoir analyser la chronologie et l'ordre des transitions pour chaque trajectoire et en déceler les "logiques internes "(Courgeau, 1999).

2. Mesurer les caractéristiques des ménages et des localités dans lesquels les individus ont vécu afin de mettre en évidence leur influence sur les comportements individuels.

3. Examiner l'effet des transformations des structures sociales, économiques, écologiques et autres sur les trajectoires individuelles.

C'est ce défi que nous avons voulu relever par la réalisation de l'enquête sur la dynamique migratoire, l'insertion urbaine et l'environnement au Burkina Faso.

Pour collecter les informations nécessaires à l'étude des stratégies de reproduction des populations du Burkina Faso, nous avons conçu une opération de collecte impliquant la réalisation d'une enquête rétrospective biographique, la réalisation d'une enquête rétrospective communautaire et l'exploitation de sources de données secondaires.

L'enquête rétrospective biographique, dont le terrain s'est terminé en juillet 2000, visait à collecter l'information nécessaire à la reconstitution des trajectoires individuelles; à l'analyse des interactions entre différentes trajectoires de la biographie des individus; et à l'étude de l'influence des caractéristiques du ménage sur les trajectoires individuelles. C'est le potentiel et l'originalité de cette enquête que nous mettrons en évidence dans la suite de cette note.

L'enquête communautaire et le recours aux sources secondaires, deux étapes qui sont en cours et qui seront achevées en 2001 , visent essentiellement à cerner les facteurs environne- 
mentaux qui, comme on l'a vu précédemment, sont susceptibles de façonner les comportements individuels, tout particulièrement en milieu rural burkinabè. En conclusion, nous reviendrons sur l'importance de ces deux volets pour l'étude des stratégies de reproduction.

\section{L'ENQUÊTE BIOGRAPHIQUE RÉTROSPECTIVE " DYNAMIQUE MIGRATOIRE, INSERTION URBAINE ET ENVIRONNEMENT AU BURKINA FASO "}

\section{Plan d'échantillonnage}

L'enquête porte sur l'ensemble du pays, qui comptait environ 10,5 millions d'habitants au recensement de 1996. Il s'agit d'une enquête stratifiée avec plan d'échantillonnage par grappe à deux degrés (" two-stage cluster sampling ".

Notre stratification se base tout d'abord sur la distinction entre le milieu urbain et le milieu rural. Nous avons retenu trois strates urbaines: Ouagadougou, la capitale; Bobo Dioulasso, l'ancienne capitale et seconde ville du pays; et Banfora, un centre secondaire ayant connu une croissance spectaculaire au cours des trente dernières années du fait de la migration rurale-urbaine. En milieu rural, nous avons identifié cinq strates sur la base de critères agro-climatiques, d'une part, et socioculturels, d'autre part. Ce découpage s'apparente, à quelques modifications près, aux cinq grandes régions définies par le ministère de l'Agriculture du Burkina (INSD, 1998).

Pour déterminer la taille de l'échantillon, nous avons pris en compte :

- la précision exigée au niveau de l'estimateur retenu, soit la proportion de personnes ayant migré au moins une fois dans leur vie;

- l'effet de grappe;

- la finesse des analyses que nous voulions mener (comparaison par milieu, sexe et génération).

Sur cette base, nous nous sommes fixé comme objectif de collecter 9188 biographies d'individus âgés de 15 à 64 ans appartenant à 4258 ménages provenant de 157 zones de dénombrement (ZD) tirés aléatoirement. Les tirages sont indépendants dans chacune des huit strates. Il s'agit donc de huit échantillons distincts. Dans chaque strate, au premier niveau, les ZD ont été tirées proportionnellement à leur taille en nombre de ménages à partir de la base de sondage du recensement 
de 1996 (mise à jour en milieu urbain). Au deuxième niveau, un nombre fixe de ménages a été tiré aléatoirement dans les $Z D$ retenues (25 ménages dans les zones rurales et 20 dans les centres urbains) ${ }^{3}$ après dénombrement du nombre total de ménage dans chacune de ces $Z \mathrm{D}$. Un questionnaire ménage a été administré à tous les ménages ainsi tirés.

Dans ces ménages, tous les membres présents et âgés de 25 à 64 ans étaient éligibles pour le questionnaire biographique individuel. Pour les membres de 15 à 24 ans, nous avons tiré de façon aléatoire une personne sur deux pour l'administration du questionnaire biographique individuel. Nous avons procédé ainsi pour tenir compte du poids relativement êlevé de ce groupe d'âge dans la population ainsi que du caractère fortement tronqué de leur biographie.

La collecte a duré quatre mois, de mars à juillet 2000. Au total, nous avons recueilli des données sur 22999 personnes appartenant à 3517 ménages et complété 8644 biographies d'individus.

\section{L'information collectêe sur les individus}

Pour la collecte des données individuelles, nous nous sommes inspirés de l'expérience de l'enquête sur l'insertion des migrants à Bamako (IMMUS-Bamako) réalisée conjointement par le CERPOD et le Département de démographie de l'Université de Montréal en 1992 (Ouédraogo et Piché, 1995; Marcoux, Piché et Konaté, 1999). Pour mieux coller aux objectifs spécifiques de notre recherche et pour tenir compte des leçons de l'enquête de Bamako, nous avons apporté certaines innovations sur lesquelles nous insisterons davantage dans la présentation qui suit.

Nous avons recueilli les informations nécessaires à la reconstitution des trajectoires résidentielle, professionnelle, conjugale et, pour les femmes, génésique. Comme pour l'enquête de Bamako, nous avons eu recours à la fiche "Âgeévénement " (AGEVEN) pour obtenir une chronologie aussi précise que possible des transitions qui jalonnent, clans différents domaines, la biographie des individus (Antoine et al., 1987). Cette fiche "permet de mieux repérer les événements dans le

3 En milieu urbain non loti, nous avons tiré 25 ménages, tout comme en milieu rural, pour tenir compte d'une taille des ménages plus faible en raison de la stratégie d'occupation de telles zones. 
temps, les uns par rapport aux autres et d'aider les enquêtés à situer temporellement des événements dont ils peuvent avoir oublié la date. [...] L'identification des événements familiaux facilite le repérage des autres événements" (Marcoux et al., 1999 : 208). La figure 1 illustre l'utilité de cette fiche à partir d'un exemple fictif.

Pour les trajectoires résidentielle et professionnelle, nous avons retenu toutes les périodes de trois mois et plus. Nous voulions ainsi etre en mesure de mieux cerner les migrations de courte durée qui, selon nous, sous-tendent les efforts de diversification des activitës dans le cadre des stratégies de reproduction des populations rurales. Le critère de six mois habituellement retenu ne nous semblait guère adapté au contexte agro-économique du Burkina, la saison sèche propice aux migrations temporaires atteignant rarement cette limite. De plus, nous pensons que le processus d'insertion des migrants en milieu urbain se caractérise par l'enchaînement de courtes périodes d'activité et de résidence qui nous auraient alors largement échappé. Cela nous a également amenés à retenir toutes les périodes de résidence d'une durée minimale de trois mois sans tenir compte du critère habituel de franchissement d'une frontière administrative. Nous pourrons ainsi, par exemple, mieux cerner les processus de mobilite intra-communautaire qui s'inscrivent dans les stratégies d'accès à l'indèpendance économique des jeunes. Au total, l'enquête a recueilli une information précise sur 26637 périodes de résidence: 44 pour cent en milieu urbain, 45 pour cent en milieu rural et 11 pour cent à l'étranger, presque toujours en Côte-d'Ivoire.

Pour étudier les modalités d'accession à la propriété en milieu urbain et ainsi mieux saisir les stratégies de constitution $\mathrm{du}$ patrimoine foncier, nous avons obtenu, pour chacune de ces périodes de résidence, le statut d'occupation des ẹquêtés et, pour les propriétaires, le mode d'obtention du terrain.

La collecte d'informations sur la multi-activité en Afrique subsaharienne, spécialement chez les femmes, se heurte souvent à de nombreux obstacles lors d'enquètes quantitatives de grande envergure. À cet égard, l'enquête de Bamako n'a pas fait exception (Marcoux et al., 1999). Plusieurs travaux de nature plus qualitative placent cependant ce phénomène au cœur des stratégies de reproduction des groupes les plus vulnérables. Nous avons donc accordé une attention particulière à la collecte des informations sur les activités. Au total, 25952 périodes d'activité ont ainsi été documentees. 


\begin{tabular}{|c|c|c|c|}
\hline & Evénements familiaux & Itinéraire résidentiel & $\begin{array}{l}\text { Activités scolaires et } \\
\text { professionnelles }\end{array}$ \\
\hline 2000 & Naiss Adioma & & S Vente \\
\hline 1999 & Naiss. Fanta & & Tient son de bois \\
\hline 1998 & Déces Salif & & propre cabaret Vannerde \\
\hline 1997 & Naiss. Salif, dc. Zénabou & & 6 \\
\hline 1996 & Naiss. Zénabou & & Alde belle-mére a \\
\hline 1995 & & & \begin{tabular}{|l|l} 
préparer le dolo & mil germé
\end{tabular} \\
\hline 1994 & Mar, Abou & Ró Soala/ Nanaro/Bulkiende & ( \\
\hline 1993 & Sép. Ali & R5 Ouaga/ Goughin sud & Employée dans un \\
\hline 1992 & (1) & & salon de colifure \\
\hline 1991 & Union Ali, naiss. Awa & R4 Ouaga/secteur 28 & Apprentie \\
\hline 1990 & Noiss Moussa & R3 Oulagg/ secterir 12 & contrinere \\
\hline 1989 & Décés Maman & & Quéte d'emploi \\
\hline 1988 & & R2 Kovtouggu & (Ftermotion des chudes) \\
\hline 1987 & & & CEPE \\
\hline \multicolumn{4}{|l|}{1986} \\
\hline \multicolumn{4}{|l|}{1985} \\
\hline 1984 & 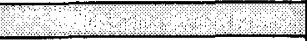 & (O) & (1) \\
\hline \multicolumn{4}{|l|}{1983} \\
\hline \multicolumn{4}{|l|}{1982} \\
\hline 1981 & & & $C P 1$ \\
\hline 1980 & 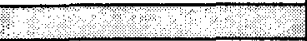 & R1 Ouagd/ secteri 68 & 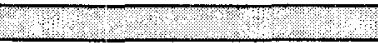 \\
\hline 1979 & Décès Papa & & \\
\hline 1978 & & (20) & से \\
\hline \multicolumn{4}{|l|}{1977} \\
\hline \multicolumn{4}{|l|}{1976} \\
\hline \multicolumn{4}{|l|}{1975} \\
\hline 1974 & Naiss. EGO & & 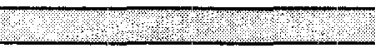 \\
\hline
\end{tabular}

FIGURE 1 - Exemple de fiche AGEVEN (individu fictif de sexe féminin)

Pour chaque période d'activité principale, notre questionnaire permettait de retranscrire jusqu'à trois activités secondaires. Nos premiers résultats indiquent que l'exercice d'au moins une activité secondaire caractérise près d'une période de travail sur cinq $(17,7$ pour cent). Toutes les activités ont été saisies en clair par les enquêteurs. À l'étape de la codification, nous avons utilisé une nomenclature des professions que nous avons enrichie de tous les " petits métiers " déclarés et qui ne s'y trouvaient pas. Enfin, tout au long de la formation et de la supervision des enquêteurs, des efforts particuliers ont porté sur le recueil des activités spécifiques des femmes et des enfants. De plus, pour chaque période d'activité déclarée, nous avons demandé au répondant s'il recevait de l'aide de la part de ses enfants afin de mieux cerner cette dimension importante des stratégies de survie des ménages les plus vulnérables.

Comme pour l'enquête de Bamako, nous avons interrogé les individus sur les filières d'accès à l'emploi pour chaque période 
d'activité afin de vérifier, notamment, l'importance des réseaux de parenté dans le processus d'insertion professionnelle.

Le module de notre questionnaire biographique portant sur l'histoire génésique des femmes a permis d'obtenir de l'information sur 17544 naissances. Cette section du questionnaire faisait une large place aux déterminants proches de la fécondité, notamment les moyens pris par les femmes avant chaque naissance, et au moment de l'enquête pour les femmes sans enfants, afin de retarder ou éviter une naissance. Nous nous sommes intéressés à toutes les méthodes, y compris les plus rudimentaires. De plus, nous avons recueilli pour chaque enfant la durée de l'allaitement exclusif et l'âge au sevrage définitif, facteurs importants de la mortalité et de la morbidité infantiles. Une section de ce module concerne spécifiquement le devenir de tous les enfants auxquels les femmes ont donné naissance. Cela nous permettra de savoir, par exemple, pourquoi ils n'ont jamais été inscrits à l'école; pourquoi ils ont arrêté et avec quels diplômes; quand ils ont quitté le domicile familial pour la première fois; pour quelle destination et pour quel motif; s'ils ont rejoint un membre de la famille à destination... En documentant des pratiques de confiage d'enfants à des fins de scolarisation, de travail, de mariage etc., les informations ainsi collectées nous permettront de mettre en évidence des aspects importants des stratégies de survie et de promotion sociale.

Les informations ainsi collectées à partir du questionnaire individuel vont nous permettre, d'une part, de reconstituer les trajectoires des individus dans différents domaines de leur histoire de vie et, d'autre part, d'analyser les interactions entre ces trajectoires. Bien qu'essentielles pour cerner les stratégies de reproduction mises en œuvre par les populations burkinabè, ces informations ne sont cependant pas suffisantes. En effet, comme on l'a vu précédemment, ces stratégies sont également façonnées dans une large mesure par les caractéristiques dynamiques de l'environnement dans lequel ces individus évoluent au cours de leur vie. Nous devons donc disposer d'informations chronologiques sur les principaux cadres de vie des individus : leur ménage, leur localité, leur région de résidence...

L'opération de collecte de données que nous avons mise en œuvre visait donc à recueillir ce type d'informations contextuelles à partir de l'enquête rétrospective en ce qui concerne le ménage et la localité et à partir des sources de données secondaires pour les niveaux supérieurs (région, pays). 


\section{L'information collectée sur le ménage}

L'information recueillie par notre enquête au niveau du ménage provient du questionnaire ménage que nous avons administré à tous les chefs des ménages sélectionnés ainsi qu'à (une de) leur(s) épouse(s). Elle provient également, et pour une large part, du questionnaire individuel.

Le questionnaire ménage nous a permis de collecter des données sur différents aspects du cadre de vie immédiat de l'individu au moment de l'enquête :

- la structure socio-démographique du ménage établie à partir des informations de la feuille ménage;

- la situation économique du ménage à partir des questions sur le patrimoine et l'équipement du ménage;

- les caractéristiques de l'habitat et de l'environnement domestique (nature des matériaux de l'habitation; sources d'énergie utilisées pour la cuisson des aliments, l'éclairage etc.; sources d'eau potable; type d'aisance; mode de gestion des ordures domestiques; etc.).

Ont également été collectées auprès du chef de ménage les données concernant les membres du ménage qui ont émigré à l'étranger : caractéristiques socio-démographiques au moment du départ, date et lieu d'émigration.

Les données collectées par le questionnaire individuel permettent d'enrichir considérablement cette information. Le premier module de ce questionnaire nous renseigne sur l'origine familiale des individus. Les questions de ce module portent notamment sur le nombre d'enfants qu'ont eu le père et la mère de l'enquêté ainsi que sur son rang de naissance parmi les enfants de son père et de sa mère. Cette information est pertinente car on peut penser qu'au Burkina Faso les modalités d'accès à l'indépendance économique dans lesquelles s'inscrit souvent la première migration varient selon la place qu'occupent les individus dans leur fratrie (Boutillier, Quesnel et Vaugelade, 1985).

Nous disposons également de l'information sur l'activité économique et le statut professionnel du père et de la mère de l'enquêté de façon à caractériser le milieu familial dont il est issu.

Le questionnaire biographique nous a en outre permis de collecter des informations très précises sur les caractêristiques de lhabitat (secteur loti ou non, types d'habitat, nature des 
matériaux...) et de l'environnement domestique (énergie, eau, évacuation des ordures...) pour chacune des résidences dans lesquelles les enquêtés auront séjourné au cours de leur vie à partir de l'âge de 6 ans. Au cours d'une période de résidence, les changements éventuels dans certains domaines de cet environnement domestique ont également été saisis et datés. Cela nous permettra ainsi de mesurer l'influence sur les trajectoires individuelles de facteurs liés aux différents aspects du cadre de vie immédiat des enquêtés.

Dans le même ordre d'idées, le questionnaire biographique nous permet de connaitre le nombre précis d'enfants que les femmes ont à charge dans leur ménage à chaque moment de leur vie. Nous disposons également de l'activité et du statut professionnel des conjoint(e)s successif(ve)s au début et à la fin de chaque union. Pour les femmes, on sait combien d'épouses avait déjà leur conjoint au moment de l'union et quand sont arrivées au cours de l'union les co-épouses successives (on connait aussi la date de la rupture éventuelle de leur mari avec ces nouvelles femmes). On peut donc retracer à partir de l'union les différents stades où la femme reçoit de moins en moins de soutien de la part de son mari.

Les informations collectées dans le questionnaire biographique individuel nous permettront enfin de cerner les interactions entre les trajectoires des conjoints résidant dans un même ménage. Il s'agit en quelque sorte de reconstituer des biographies de couples à partir d'une clé introduite dans les questionnaires biographiques respectifs des conjoints. Cela nous permettra, par exemple, d'estimer l'effet de la migration du mari sur l'activité économique de son épouse. On entrevoit ainsi toutes les possibilités qui s'offriront à l'analyse.

\section{CONCLUSION}

Pour cerner les stratégies de reproduction mises en œuvre au Burkina Faso, la réalisation d'une enquête biographique rétrospective sur un échantillon représentatif au plan national s'imposait. Les informations ainsi recueillies permettront en effet de reconstituer les trajectoires des individus dans des domaines importants de leur histoire de vie. Elles rendent également possible l'analyse des interactions complexes entre ces différentes trajectoires. Elles permettront enfin de mesurer l'influence de caractéristiques importantes du cadre de vie im- 
médiat des individus sur leurs trajectoires résidentielles, professionnelles et familiales.

Si de telles informations sont nécessaires pour l'étude des stratégies de reproduction, elles ne sont cependant pas suffisantes. En effet, l'influence des facteurs opérant à des niveaux supérieurs sur les comportements individuels doit également être prise en compte. Nous pensons par exemple aux caractéristiques des localités de résidence des individus; ou encore à des facteurs environnementaux opérant à une échelle régionale; et enfin à des facteurs liés à la mise en ceuvre de politiques nationales dans différents domaines.

L'intégration de tels facteurs dans des modèles dynamiques est maintenant possible sur le plan méthodologique grâce aux avancées récentes en statistique. Il restait à surmonter les problèmes d'observation et de données disponibles signalés par Courgeau et Bacaïni (1997). C'est pourquoi notre recherche s'appuie non seulement sur l'enquête biographique rétrospective, mais aussi sur deux autres sources de données. Il s'agit d'abord des données qui seront collectées dans le cadre d'une enquete communautaire également rétrospective. Ce volet de la recherche est sans doute le plus original. Jusqu'ici, peu d'enquêtes ont collecté des données sur les caractéristiques communautaires et, sauf de rares exceptions (Axinn, Barber et Ghimire, 1997), il s'agissait des caractéristiques des communautés de résidence des répondants au moment de l'enquête.

Notre enquête communautaire portera sur toutes les localités (c'est-à-dire le village en milieu rural et le quartier en milieu urbain) dans lesquelles aura résidé l'ensemble de nos répondants au cours de leur vie. Des questionnaires spécifiques au milieu rural et au milieu urbain nous permettront de recueillir des informations sur l'historique des infrastructures sanitaires, scolaires, économiques, religieuses et culturelles, sur les projets de développement qui ont été réalisés dans la localité, sur les catastrophes (sécheresses, famines, épidémies, etc.) survenues au cours des cinquante dernières années, sur l'évolution des voies d'accès à la localitê, et, en milieu rural, sur la dynamique des structures foncières.

Pour réunir l'information sur les facteurs opérant à un niveau supérieur, nous exploiterons des sources de données secondaires. Il s'agit par exemple des séries chronologiques concernant les précipitations, la dégradation des sols, les statistiques agricoles de production, de rendements et de superficie. Ces différentes séries, plus ou moins longues, portent sur 
des niveaux plus ou moins fins de découpage géographique : le département, la province, la région.

Notre démarche constitue ainsi une première tentative d'envergure de mise en oeuvre "des systèmes d'observation représentatifs des contextes sociaux diversifiés et hiérarchisés" (Loriaux, 1987 : 364), nécessaire à la compréhension des stratégies de reproduction des populations sahéliennes.

\section{RÉFÉRENCES BIBLIOGRAPHIQUES}

ANCEY, G. 1983. Monnaie et structure d'exploitation en pays Mossi. Ouagadougou et Paris, ORSTOM, $240 \mathrm{p}$.

ANTOINE, P., X. BRY et P. D. DIOUF. 1987. "La fiche AGEVEN : un outil pour la collecte des données rétrospectives", Techniques d'enquêtes, 13, 2 : 173-181.

AXINN, W. G., J. S. BARBER et D. J. GHIMIRE. 1997. "The neighborhood history calendar: A data collection method designed for dynamic multilevel modeling", Sociological Methodology, 27 : 355392.

BALAN, J., H. L. BROWNING, E. JELIN et L. LITZLER. 1969. " A Computerized Approach to the Processing and Analysis of Life Histories Obtained in Sample Surveys ", Behavioral Science, 14 : 105-115.

BLOSSFELD, H. P. 1996. "Macro-sociology, rational choice theory, and time. A theoretical perspective on the empirical analysis of social processes ", European Sociological Review, 12, 2 : 181-206.

BLOSSFELD, H. P., E. KLIJZING, K. POHL et G. ROHWER. 1999. "Why do cohabiting couples marry? An example of a causal event history approach to interdependent systems ", Quality and Quantity, $33: 229-242$.

BOURDIEU, P. 1994. "Stratégies de reproduction et modes de domination ", Actes de la recherche en sciences sociales, no $105: 3-12$.

BOUTILLIER, J.-L. 1975. "Données économiques concernant les migrations de la main d'œuvre voltaïque ", dans Les Migrations de travail Mossi : évolution récente et bilan des migrations de travail. Les migrants et la société Mossi. (c) Ministère du Travail et de la Fonction publique Haute-Volta, Fascicule 1: 147-203.

BOUTILLIER, J.-L., A. QUESNEL et J. VAUGELADE. 1985. "La migration de la jeunesse au Burkina ", Les Cahiers de l'ORSTOM, Série Sciences humaines, 21, 2-3:243-249.

CORDELL, D. D., J. W. GREGORY et V. PICHÉ. 1987. "African historical demography. The search for a theoretical framework n, dans D. D. Cordell et J.W. Gregory, éd. African Population and Capitalism: Historical Perspectives. Boulder et Londres, Westview Press : 14-32. 
CORDELL, D. D., J. W. GREGORY et V. PICHÉ. 1996. Hoe and Wage. A Social History of Circular Migration System in West Africa. Boulder, Westview : $384 \mathrm{p}$.

COURGEAU D., 1999. "Introduction ", dans Groupe de réflexion sur l'approche biographique. Biographies d'enquêtes. Méthodes et savoirs. Paris, INED.

COURGEAU, D., et B. BAÏCCANI. 1997. "Analyse multi-niveaux en sciences sociales ", Population, 52, 4 : 831-863.

COURGEAU, D., et É. LELIÉVRE. 1993. "Nouvelles perspectives de l'analyse biographique ", Cahiers québécois de démographie, 22, 1 : 23-44.

DENIEL, R. 1968. De la savane à la ville. Essai sur les migrations des Mossi vers Abidjan et sa Région. Collection des travaux du CASHA, $185 \mathrm{p}$.

GERVAIS, R. 1990. Population et politiques agricoles coloniales dans le Mossi. 1919-1940. Universite Paris 7, thèse de doctorat.

GRUENAIS, M.-E. 1985. "Aînés, aînées; cadets, cadettes. Les relations aînés/cadets chez les Mossi du Centre (Burkina Faso) ", dans M. ABELES et C. COLLARD, èd., Âge, pouvoir et société en Afrique noire. Paris, Karthala : 219-245.

GUIELLA, G., et J. POIRIER. 2000. "Fondements socio-économiques de la fécondité chez les Mossi du plateau central (Burkina Faso) ", dans M. PILON et A. GUILLAUME, éd. Maitrise de la fécondité et planification familiale au Sud. Paris, IRD Éditions : 93-110.

HAMPSHIRE, K., et S. RANDALL. 1999. "Seasonal labour migration strategies in the Sahel: Coping with poverty or optimising security? ", International Journal of Population Geography, 5, 5: 367-385.

INSD (Institut national de la statistique et de la démographie du Burkina Faso). 1998. Enquête prioritaire 2 - Plan de sondage. Ouagadougou.

IZARD, M. 1985. Le Yatenga précolonial. Un ancien royaume du Burkina. Paris, Karthala, 164 p.

KEVANE, M., et B. WYDICK. 2001. "Social norms and the time allocation of women's labor in Burkina Faso ", Review of Development Economics, 5, 1.

KEVANE, M., et L. GRAY. 1999. "A woman's field is made at night: Gendered land rights and norms in Burkina Faso ", Feminist Economics, 5, 3.

LAURENT, P.-J. 1996. "Dynamiques matrimoniales chez les Mossi Burkina Faso ", dans Association belge des africanistes, éd. Phénomènes informels et dynamiques culturelles en Afrique.

LELIËVRE, E. 1999. "Collecter des données de mobilitè : des histoires migratoires aux biographies d'entourage ", Espace, Populations, Sociétés, 2 : 195-205. 
LORIAUX, M. 1987. "L'analyse contextuelle : renouveau théorique ou impasse méthodologique ", dans J. DUCHENE, G. WUNSCH et $\dot{E}$. VILQUIN, éd. L'Explication en sciences sociales : la recherche des causes en démographie. Louvain-la-Neuve, Ciaco : 333-368.

MARCOUX, R., V. PICHÉ et M. KONATÉ. 1999. "L'enquête Insertion des migrants en milieu urbain au Sahel ", dans Biographies d'enquêtes. Paris, PUF : 203-220.

MCMILLAN, D. E., J. H. SANDERS, D. KOENIG, K. AKWABI-AMEYAW et T. M. PAINTER. 1998. "New land is not enough : Agricultural performance of new lands settlement in West Africa ", World Development, 26, $2: 187-211$.

MITTERAUER, M. 1992. "Peasant and non-peasant family forms in relation to the physical environment and the local economy", Journal of Family History, 17, 2 : 139-159.

MOEN, P., et E. WETHINGTON. 1992. "The concept of family adaptive strategies ", Annual Review of Sociology, $18: 233-251$.

OUÉDRAOGO, D., et V. PICHÉ. 1995. L'Insertion urbaine à Bamako (Mali). Paris, Karthala, 206 p.

PICHÉ, V., et J. POIRIER. 1990. "Les théories de la transition démographique : vers une certaine convergence? ", Sociologie et Sociétés, 22, $1: 179-192$.

PICHÉ, V., et J. POIRIER. 1995. "Les approches institutionnelles de la féconditè ", dans H. GÉRARD et V. PICHÉ, éd. La Sociologie des populations. Les Presses de l'Université de Montréal, AUPELF/ UREF : 117-138.

POIRIER, J., et G. GUIELLA. 2000. "Fondements socio-économiques de la fécondité chez les Mossi du plateau central (Burkina Faso) ", dans M. PILON et A. GUILlAUME, èd. Maitrise de la fécondité et planification familiale au Sud. Paris, IRD Éditions : 93-110.

POIRIER, J., V. PICHÉ et G. NEILL. 1989. "Travail des femmes et fécondité dans les pays en développement: que nous a appris l'enquête mondiale de la fécondité?", Cahiers québécois de démographie, 18, 1 : 159-185.

POOL, I., et S. COULIBALY. 1970. Demographic Transition and Cultural Continuity in the Sahel: Aspects of the Social Demography of Upper Volta. Cornell University, $237 \mathrm{p}$.

REARDON, T., et J. E. TAYLOR. 1996. "Agroclimatic shock, income inequality, and poverty: Evidence from Burkina Faso ", World Development, 24, 5 : 901-914.

REARDON, T., P. MATLON et C. DELGADO. 1988. "Coping with household-level food insecurity in drought-affected areas of Burkina Faso ", World Development, 16 : 1044-1075.

SAINT-PIERRE, M.-H., J. W. GREGORY et A. SIMMONS. 1986. "Structure dêmographique des ménages et comportement migratoire en Haute-Volta (Burkina Faso) ", dans D. GAUVREAU, J. W. 
GREGORY, M. KEMPENEERS et V. PICHÉ, éd., Démographie et sous-développement dans le Tiers-Monde. Montréal, Université McGill, Centre for Developing Area Studies : 111-142.

SAUERBORN, R., A. M. ADAMS et M. HIEN. 1996. "Household strategies to cope with the economic costs of illness ", Social Science and Medicine, 43, 3: 291-301.

SAWADOGO, K., et S. LARIVIĖRE. 1993. Caractéristiques socio-économiques et stratégies des ménages en matière de sécurité alimentaire dans la province du Passoré, CEDRES/Laval, Ouagadougou.

SINGH, R. D. 1988. Economics of the Family and Farming Systems in Sub-saharan Africa. Boulder, Westview Press, $210 \mathrm{p}$.

SKINNER, E. P. 1965. "Labor migration among the Mossi of the Upper-Volta ", dans H. KUPER, éd. Urbanisation and Migration in West Africa, Berkeley, Los Angeles, University of California : 60-84.

UPCHURCH, D. M., L. A. LILLARD et C. W. PANIS. 1995. Updating Women's Life Course: Theoretical and Methodological Considerations. Communication présentee au Congrès annuel de la Population Association of America, San Francisco, California, 68 avril.

\begin{abstract}
Jean POIRIER, Victor PICHÉ, Gaël LE JEUNE, Bonayi DABIRÉ and Hamdou Rabby WANE

\section{A RESEARCH PROJECT ON THE REPRODUCTIVE STRATEGIES OF POPULATIONS IN THE SAHEL BASED ON THE SURVEY "MIIGRATION DYNAMICS, URBAN INTEGRATION AND ENVIRONMENT IN BURKINA FASO}

This paper discusses the national survey "Migration Dynamics, Urban Integration and Environment in Burkina Faso" conducted in 2000-2001 by the Demography Department of the Université de Montréal, CERPOD in Bamako and UERD in Ouagadougou. The first section discusses the conceptual background of the survey aiming to provide data on the various reproductive strategies adopted by the populations of Burkina Faso. The second section focuses on the objectives and design of the survey and describes the information collected at the different levels of analysis: individuals, households, communities, regions, and the country as a whole. For each level, methodological issues of data collection and analysis are discussed.
\end{abstract}

